\title{
Noise Effect Reduction on a MEMS Based AC Voltage Reference Source Using Artificial Neural Network
}

\author{
Samane Sadat Hashemipour ${ }^{1}$, Amir Abolfazl Suratgar ${ }^{2,3 *}$, Hamid Hoseini ${ }^{4}$ \\ ${ }^{1}$ M.Sc. Student, Department of Electrical Engineering, Arak A. University, Arak, Iran. \\ ${ }^{2}$ Assistant Professor, Department of Electrical Engineering, Arak University, Arak, Iran. \\ ${ }^{3}$ Assistant Professor, Department of Electrical Engineering, Amirkabir University \\ of Technology (Tehran Polytechnic), Tehran, Iran. \\ ${ }^{4}$ Assistant Professor, Department of Electrical Engineering, \\ Arak A. University, Arak, Iran. \\ *a-suratgar@araku.ac.ir, TEL: +98-861-223-2813, FAX:+98-861-222-5946.
}

\begin{abstract}
This paper presents a new method in order to reduce noise effect in an AC voltage reference source. The AC voltage reference source is implemented on MEMS technology. It uses capacitive MEMS technology. The reference is based on the characteristic AC current-voltage curve MEMS component. The multilayer neural network is used. The neural network (NN) uses the Levenberg-Marquardt (LM) method for training. The noise effect on an electronic circuit is investigated. The simulation results are very promising.
\end{abstract}

Keywords: AC voltage reference source noise effect reduction, MEMS, Neural Network

\section{RESUMEN}

El presente trabajo presenta un método nuevo para reducir el efecto de ruido en una referencia de fuente de voltaje de AC. La referencia de fuente de voltaje se implementa mediante tecnología MEMS; emplea tecnología capacitiva MEMS. La referencia se basa en la curva de corriente-voltaje de CA característica del componente MEMS. Se utiliza la red neuronal multicapas. La red neuronal (RN) usa el método Levenberg-Marquardt (LM) con fines de experimentación. Asimismo se investiga el efecto de ruido en un circuito electrónico. Los resultados de simulación son muy prometedores.

\section{Introduction}

There are only two voltage references, which are fundamentally based on $A C$ voltage, namely the $A C$ voltage Josephson reference and the MEMSbased AC voltage reference. In this paper, we will discuss the MEMS-based AC voltage reference. Many researchers have worked in this area of research. M. Suhonen et al. presented an AC voltage reference based on a capacitive microelectromechanical system (MEMS) [1]. They described micromechanical AC and DC standards suitable for compact, low-cost precision electronics applications. The standards are based on controlling the charge of a parallel-movingplate capacitor. They showed the basic principle of the $A C$ and $D C$ standards and preliminary experiments with the AC voltage standard. Design and characterization of a high stability capacitive MEMS device intended for an AC voltage reference at $100 \mathrm{kHz}$ or higher frequencies is

presented by A. Karkkainen et al [2]. They presented an AC root-mean-square (RMS) voltage reference based on MEMS component in 2005[3]. In their work, the device stability was investigated through various experiments.

They also investigated the stability of microelectromechanical devices for electrical metrology [4]. Such devices are formed from micromachined electrodes of which at least one is supported by a compliant structure such that an electrostatic force between two electrodes displaces the moving electrode. The properties of these electromechanical devices can be very stable if they are fabricated from single-crystalline silicon and sealed hermetically in a low-pressure atmosphere. In comparison to several semiconducting reference devices, micromechanical components are large in size and consume a 
negligible power. Thus, a low $1 / \mathrm{f}$ noise level is expected. Erik F. Dierikx presented two AC voltage references in [5]. H. Seppa investigated applications of microsystems in precision measurements [6]. R. F. Wolffenbuttel and van C. J. Mullem discussed on the relationship between microsystems technology and metrology in [7]. Dynamic and electrical analysis of MEMS capacitor with accelerated motion effects was investigated by K. Kawano et al in [8]. M. Behera et al presented accurate simulation of phase noise in RF MEMS VCOs in [9].

\section{An overview on MEMS-based AC voltage reference source}

Voltage references are fundamental building blocks in many instruments like data logging systems, digital multimeters, and calibrators. The operation of the MEMS-based AC voltage reference is based on a characteristic property of an electrostatic MEMS component: the pull-in voltage. The component is actuated to the maximum point of the characteristic currentvoltage curve using $A C$ current. The maximum voltage depends only on the component geometry and material properties and it is therefore an excellent reference. The benefits of MEMS components in reference applications are good stability, low $1 / \mathrm{f}$ noise, large operation voltage range, small size, and low power consumption. We first investigated the properties of the MEMS capacitor. The moving plate capacitor, shown in Figure 1 , is actuated to the maximum point of its characteristic current-voltage curve using $A C$ current $I(t)=\hat{I} \sin \omega t$. There is also an effective $D C$ voltage, $V_{D C}$, over the component due to the built-in voltage and charges in the oxide layer. The electrostatic force $F_{E}$ attracts the electrodes together while the spring force $F_{M}$ due to the elastically suspended electrode, tries to restore the plate position. The force balance equation is

$$
F_{E}=F_{E, I}+F_{E, V}=-\frac{d}{d x}\left(E_{I}+E_{V}\right)=k x=F_{M}
$$

where $F_{E, I}$ and $F_{E, V}$ are the electrostatic forces due to the AC current $I$ and due to the DC voltage $V_{D C}$, $E_{l}$ and $E_{V}$ are the electrostatic energies, respectively, $x$ is the deflection of the moving electrode, and $k$ is the spring constant.
Assuming that the electrodes are in parallel and the movement is translational, $F_{E, I}$ can be calculated from

$$
F_{E, I}=-\frac{d}{d x}\left(\frac{q^{2}(t)}{2 C}\right)=\frac{\hat{I}^{2}}{2 \varepsilon A \omega^{2}} \cos ^{2}(\omega t)
$$

where $C=\varepsilon A /(d-x)$ is the component capacitance, $\varepsilon$ is the permittivity of the medium, $A$ is the electrode area, $d$ is the gap between the electrodes, and $\mathrm{q}(\mathrm{t})=\int \hat{I} \sin (\omega t) d t$ is the capacitor charge. The force due to the DC voltage is

$$
F_{E, V}=-\frac{d}{d x}\left(\frac{C V^{2}{ }_{D C}}{2}\right)=\frac{1}{2} V_{D C}^{2} \frac{\varepsilon A}{(d-x)^{2}}
$$

When either the frequency of the actuation current, $\omega / 2 \pi$, is much higher than the component mechanical resonance frequency or the motion of the moving electrode is sufficiently damped, the position of the moving electrode is stationary over the period of driving frequency and the $\cos ^{2}(\omega t)$ term equals $1 / 2$.

The rms voltage $V_{A C}$ across the plate as a function of the displacement $x$ can now be calculated from Equations (1)-(3) as follows:

$$
V_{A C}=\frac{\hat{I}(d-x)}{\sqrt{2} \omega \varepsilon A}=\sqrt{\frac{2 k}{\varepsilon A}(d-x)^{2} x-V^{2}{ }_{D C}} .
$$

The maximum of $V_{A C}(\hat{I})$, which is the reference voltage, can be calculated from $d V_{A C}(\hat{I}) / d \hat{l}=(d V$ $\left.{ }_{A C}(x) / d x\right)(d x / d \hat{l})=0$.

Since $d x / d \hat{l} \neq 0$, the maximum is reached when $d V_{A C}(x) / d x=0$.

Which occurs $\hat{I}=(3 / \sqrt{2}) \omega \varepsilon V_{p i} / d$, corresponding to $x=d / 3$. The maximum of $V_{A C}$ is

$$
V_{A C}^{\max }=\sqrt{V_{p i}^{2}-V_{D C}^{2}}
$$




$$
V_{p i}=\sqrt{\frac{8 k d^{3}}{27 \varepsilon A}}
$$

is the component pull-in voltage.

Variations of $V_{D C}$ in a short period of time, $\Delta V_{D C}$ of the $V_{D C}$ can be described as $V_{D C}=V_{D C}, 0+\Delta V_{D C}$, where $V_{D C}, 0$ is a constant. Since $V_{D C} \ll V_{A C}$, Equation (5) can be expressed as [10]

$$
V_{A C}^{\max }=V_{p i}-\frac{V_{D C, 0}^{2}}{2 V_{p i}}-\frac{V_{D C, 0}}{V_{p i}} \Delta V_{D C} .
$$

Equation (7) demonstrates that selecting $V_{D C, 0}=0$, small changes in the $D C$ voltage, $\Delta V_{D C}$, have no effect on the reference voltage in the first order. A schematic view of the MEMS moving plate capacitor and its electric equivalent circuit are displayed in Figure 1.

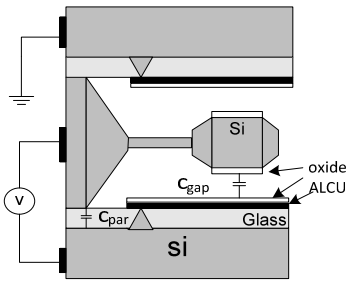

a)

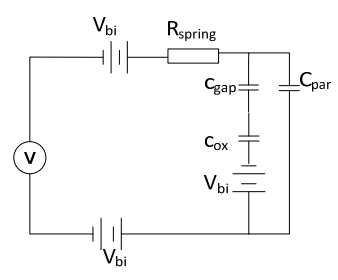

b)
Figure 1. a) Schematic structure of the silicon cantilever used as a moving plate capacitor and $b$ ) the equivalent circuit. $V$ is the external voltage source, $V_{b i}$ is the built-in voltage of the metal-silicon junction. $\mathrm{C}_{\text {gap }}$ is the component capacitance (work capacitance), which has a parasitic capacitance Cpar in parallel. $\mathrm{C}_{\mathrm{ox}}$ is the capacitor formed by the dielectric layer (usually $\mathrm{SiO} 2$ ). $\mathrm{R}_{\text {spring }}$ is the resistance of the silicon spring.

\section{Using MEMS devices in a micro electronic circuit as AC voltage reference}

The AC voltage reference electronics block diagram is show in Figure 2. A $10 \mathrm{kHz}$ AC voltage, $V_{a c-i n}$ is fed to the inverting input of the main operational amplifier OA2, which acts as a voltage to current converter. The amplitude of the input current is slowly varied over the maximum point and the maximum output voltage was recorded as the $A C$ reference voltage (the $A C$ component of $V_{\text {out }}$ in Figure 2. The instrumentation amplifier IA1 isolates the AC source. The stability of the $\mathrm{OA}_{2}$ gain is recorded at the output of the unity gain buffer amplifier $\mathrm{OA}_{3}[3]$.

The operational amplifier OA2 has a feedback so the transfer function $\mathrm{OA} 2$ is calculated as Figure 3 shows.

The transfer equation is given in Equation (8):

$$
V_{\text {OUT }}=-a V_{A}
$$

The node voltage is described by Equation (9), and Equation (10) is obtained by combining Equations (8) and (9).

$$
\begin{aligned}
& V_{A}=\frac{V_{I N} Z_{F}}{Z_{G}+Z_{F}}+\frac{V_{\text {OUT }} Z_{G}}{Z_{G}+Z_{F}} \text { for } \mathrm{I}_{\mathrm{B}}=0 \\
& \frac{V_{\text {OUT }}}{V_{I N}}=\frac{\frac{-a Z_{F}}{Z_{G}+Z_{F}}}{1+\frac{a Z_{G}}{Z_{G}+Z_{F}}}
\end{aligned}
$$

We have simulated circuit given in figure 2 with using equation (10) with replacing parameters:

$$
\mathrm{Z}_{\mathrm{F}}=\mathrm{R}_{3} \| \frac{1}{C_{M E M S}(j \omega)} \quad, \quad \mathrm{Z}_{\mathrm{G}}=\mathrm{R}_{1}
$$

where || means parallel devices.

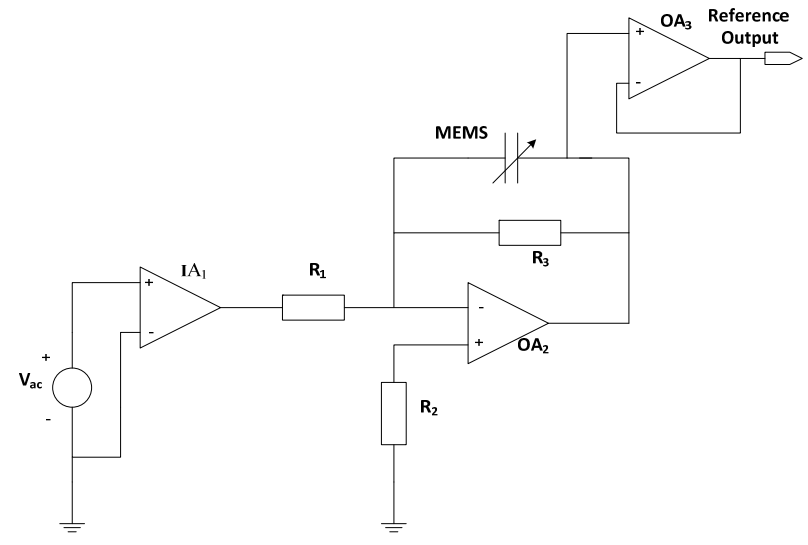

Figure 2. Electronics block diagram of the $A C$ voltage reference. 


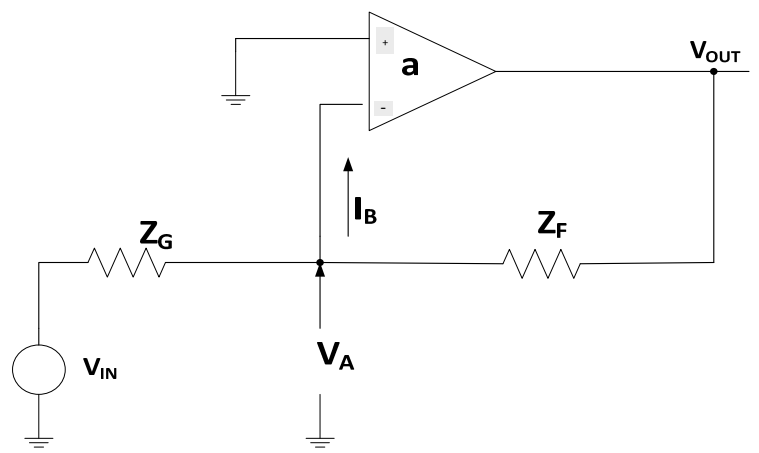

Figure 2. Inverting Op Amp.

\section{An overview of artificial neural networks}

Artificial neural networks are directly inspired from the biology of the human brain, where billions of neurons are interconnected to process a variety of complex information. We used a 1-2-1 artificial neural network (figure 4).

For training the network we suggest the Levenberg-Marquardt (LM) algorithm. In the following the Levenberg-Marquart method is reviewed [12]. In the EBP algorithm, the performance index $F(w)$ to be minimized is defined as the sum of squared errors between the target outputs and the network's simulated outputs, namely:

$$
F(w)=e^{T} e
$$

where consists of all weights of the network, $e$ is the error vector comprising the error for all the training examples. When training with the LM method, the increment of weights $\Delta \mathrm{w}$ can be obtained as follows:

$$
\Delta w=\left[J^{T} J+\mu\right]^{-1} J^{T} e
$$

Where $J$ is the Jacobian matrix, $\mu$ is the learning rate which is to be updated using the $\beta$ depending on the outcome. In particular, $\mu$ is multiplied by decay rate $\beta(0<\beta<1)$ whenever $F(w)$ decreases, whereas $\mu$ is divided by $\beta$ whenever $F(w)$ increases in a new step.
The standard LM training process can be illustrated in the following pseudo-codes,

1. Initialize the weights and parameter $\mu(\mu=.01$ is appropriate).

2. Compute the sum of the squared errors over all inputs $F(w)$.

3. Solve (8) to obtain the increment of weights $\Delta w$

4. Recompute the sum of squared errors $F(w)$

Using $\mathrm{w}+\Delta \mathrm{w}$ as the trial $\mathrm{w}$, and judge

IF trial $F(w)<F(w)$ in step 2 THEN

$$
\begin{gathered}
w=w+\Delta w \\
\mu=\mu \cdot \beta(\beta=.1)
\end{gathered}
$$

ELSE

Go back to step 2

END

$$
\mu=\mu / \beta
$$

go back to step 4

Considering the performance index is $F(w)=e^{T} e$ using the Newton method we have as

$$
\begin{aligned}
& W_{K+1}=W_{K}-A_{K}^{-1} \cdot g_{K} \\
& A_{k}=\left.\nabla^{2} F(w)\right|_{w=w_{k}} \\
& g_{k}=\left.\nabla F(w)\right|_{w=w_{k}} \\
& {[\nabla F(w)]_{j}=\frac{\partial F(w)}{\partial w_{j}}} \\
& =2 \sum_{i=1}^{N} e_{i}(w) \cdot \frac{\partial e_{i}(w)}{\partial w j}
\end{aligned}
$$

The gradient can write as

$$
\nabla F(x)=2 J^{T} e(w)
$$


Where

$$
J(w)=\left[\begin{array}{l}
\frac{\partial e_{11}}{\partial w_{1}} \frac{\partial e_{11}}{\partial w_{2}} \cdots \cdots \frac{\partial e_{11}}{\partial w_{N}} \\
\frac{\partial e_{21}}{\partial w_{1}} \frac{\partial e_{21}}{\partial w_{2}} \cdots \cdots \frac{\partial e_{21}}{\partial w_{N}} \\
\vdots \\
\frac{\partial e_{K P}}{\partial w_{1}} \frac{\partial e_{K P}}{\partial w_{2}} \cdots \cdots \frac{\partial e_{K P}}{\partial w_{N}}
\end{array}\right]
$$

$J(w)$ is called the Jacobian matrix.

Next we want to find the Hessian matrix. The $k$, j elements of the Hessian matrix yields as

$$
\begin{aligned}
{\left[\nabla^{2} F(w)\right]_{k, j} } & =\frac{\partial^{2} F(w)}{\partial w_{k} \partial w_{j}} \\
= & 2 \sum_{i=1}^{N}\left\{\frac{\partial e_{i}(w)}{\partial w_{k}} \frac{\partial e_{i}(w)}{\partial w_{j}}\right. \\
& \left.+e_{i}(w) \cdot \frac{\partial^{2} e_{i}(w)}{\partial w_{k} \partial w_{j}}\right\}
\end{aligned}
$$

The Hessian matrix can then be expressed as follows:

$$
\begin{aligned}
& \nabla^{2} F(W)=2 J^{T}(W) \cdot J(W)+S(W) \\
& S(w)=\sum_{i=1}^{N} e_{i}(w) \cdot \nabla^{2} e_{i}(w)
\end{aligned}
$$

If we assume that $S(w)$ is small, we can approximate the Hessian matrix as:

$$
\nabla^{2} F(w) \cong 2 J^{T}(w) J(w)
$$

Using (14) and (21) we obtain the Gauss-Newton method

$$
\begin{aligned}
& W_{k+1}= \\
& W_{k}-\left[2 J^{T}\left(w_{k}\right) \cdot J\left(w_{k}\right)\right]^{-1} 2 J^{T}\left(w_{k}\right) e\left(w_{k}\right) \\
& \cong W_{k}-\left[J^{T}\left(w_{k}\right) \cdot J\left(w_{k}\right)\right]^{-1} J^{T}\left(w_{k}\right) e\left(w_{k}\right)
\end{aligned}
$$

The advantage of the Gauss-Newton Method is that it does not require calculation of second derivatives. On the other hand, the Gauss-Newton method has a drawback: matrix $\mathrm{H}=\mathrm{J}^{\top} \mathrm{J}$ may not be invertible. This can be overcome by using the following modification:

The Hessian matrix can be written as

$$
G=H+\mu I
$$

Suppose that the eigenvalues and eigenvectors of $H$ are $\{\lambda 1, \lambda 2, \ldots \ldots, \lambda n\}$ and $\{z 1, z 2, \ldots \ldots . ., z n\}$, then

$$
\begin{aligned}
G z_{i}= & {[H+\mu I] z_{i} } \\
& =H z_{i}+\mu z_{i} \\
& =\lambda_{i} z_{i}+\mu z_{i} \\
& =\left(\lambda_{i}+\mu\right) z_{i}
\end{aligned}
$$

Therefore, the eigenvectors of $G$ are the same as the eigenvectors of $\mathrm{H}$, and the eigenvalues of $\mathrm{G}$ are $(\lambda i+\mu)$. Matrix $G$ is positive definite by increasing $\mu$ until $(\lambda i+\mu)>0$ for all $I$, hence the matrix will be invertible.

This leads to the Levenberg-Marquardt algorithm:

$$
\begin{aligned}
& w_{K+1}=w_{K}-\left[J^{T}\left(w_{K}\right) J\left(w_{k}\right)+\mu I\right]^{-1} J^{T}\left(w_{K}\right) e\left(w_{K}\right) \\
& \Delta w_{K}=\left[J^{T}\left(w_{K}\right) J\left(w_{K}\right)+\mu I\right]^{-1} J^{T}\left(w_{K}\right) e\left(w_{K}\right)
\end{aligned}
$$

As known, the learning parameter $\mu$ is illustrator of steps of actual output movement to desired output. In the standard LM method, $\mu$ is a constant number.

In this paper, the input matrix for training $\mathrm{NN}$ has $1^{*} 1000$ dimensions and the output matrix has $1{ }^{*} 1000$ dimensions. After training NN, for testing $\mathrm{NN}$ a $1 * 100$ matrix is given as input of $\mathrm{NN}$ and the results are evaluated. 


\section{Noise Effect Reduction on a MEMS Based AC Voltage Reference Source Using Artificial Neural Network, Samane Sadat Hashemipour et al., 49-56}

\section{Simulation results}

In this study, we have added noise with different magnitude to input voltage in node 1 in Figure 2 and eliminate noise effect with changing of R1. We used an 1-2-1 artificial neural network (Figure 4 ). The net used was feed-forward neural network trained by the Levenberg-Marquart algorithm. Mean squared error (MSE) is shown in Figure 5. Figure 6 compares the variation maximum voltage with change noise with neural network and without neural network.

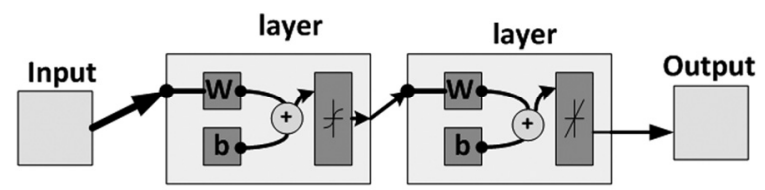

Figure 4. Schematic of neural network.

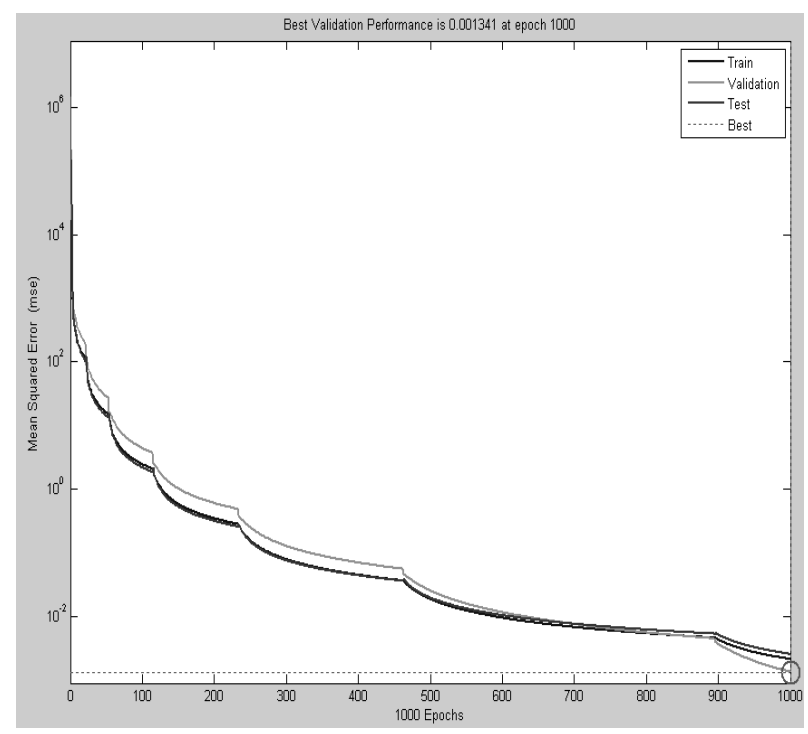

Figure 5. MSE of artificial neural network training error.

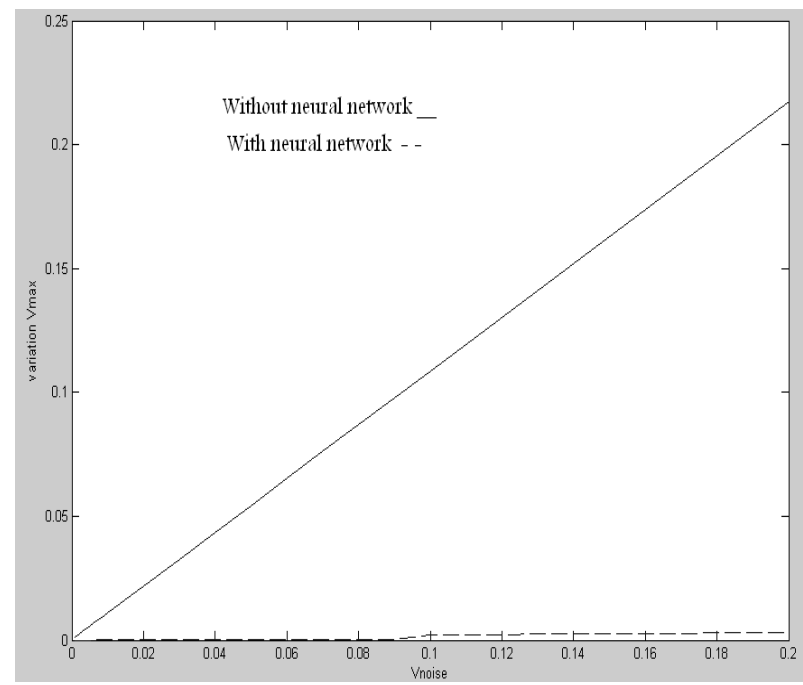

Figure 6. Variation maximum voltage with change noise with neural network and without neural network.

\section{Conclusions}

In this study, we used a neural network to decrease the effect noise on an ac voltage reference. The reference is based on the characteristic AC current - voltage curve of the component having a maximum, the value of which depends on the geometry of the component and material properties of single crystalline silicon. Stability of AC voltage reference is very important. We should try to decrease effect noise and temperature on this references so using neural network is a suitable way to compensate these effects. 


\section{References}

[1] M. Suhonen, H. Seppä, A.S. Oja, M. Heinilä, and I. Näkki, AC and DC Voltage Standards Based on Silicon Micromechanics, CPEM'98 Conf. Dig., 1998, pp. 23-24.

[2] A. Karkkainen, N. Pesonen, M. Suhonen, J. Kyynarainen, A. Oja, A. Manninen, N. Tisnek, H. Seppa, AC Voltage Reference Based on a Capacitive Micromechanical Component, IEEE Trans. June 2004, pp. 119-120

[3] A. Kärkkäinen, N. Pesonen, M. Suhonen, A. Oja, A. Manninen, N. Tisnek, and $H$. Seppä, MEMS based AC Voltage Reference, IEEE Trans. Instrum. Meas. 54, Apr. 2005, June 2004, pp. 595-599.

[4] J. Kyynäräinen, A. S. Oja, and H. Seppä, Stability of Microelectromechanical Devices for Electrical Metrology, IEEE Trans. Instrum. Meas. 50, 2001, pp. 1499-1503.

[5] Erik F. Dierikx, A MEMS-Stabilized AC Voltage Reference Source, IEEE Trans, VOL. 56, NO. 2, APRIL 2007

[6] H. Seppa, Applications of Microsystems in precision measurements, IEEE conf,June 2004 Page(s):677 - 677.

[7] R.F. Wolffenbuttel.; C.J. van Mullem, The relationship between microsystem technology and metrology, IEEE Trans, Dec 2001 Page(s):1469 - 1474.

[8] K. Kawano.;S. Shahrani.;T. Mori.;M. Kuroda.;M.M. Tentzeris, Dynamic and electrical analysis of MEMS capacitor with accelerated motion effects, , IEEE conf, April 2005 Page(s): 759 - 762.

[9] Behera, M.; Kratyuk, V.; Yutao Hu; Mayaram, K. Accurate simulation of phase noise in RF MEMS VCOs,IEEE, Proceedings of the 2004 International Symposium on Volume 3, Issue , 23-26 May 2004 Page(s): III - 677-80 Vol.3.

[10] A. Kärkkäinen, N. Tisnek, A. Manninen, N. Pesonen, A. Oja, and H. Seppä, Electrical stability of a MEMSbased AC voltage reference, Elsevier,2005.

[11] S. Haykin, Neural Networks, A Comprehensive Foundation, Prentice Hall, 1999.

[12] M. T. Hagan, H.B.Demuth, M. Beale,Pws.Publishing 1996.

[13] A. Kärkkäinen, A. Oja, J. Kyynäräinen, H. Kuisma, and $H$. Seppä, Stability of Electrostatic Actuation of MEMS, Physica Scripta, T114, 2004, pp. 193-194.
[14] S.M. Sze, Semiconductor devices, John Wiley \& Sons Ltd, 2nd edition, 2002,ISBN 0-471-33372-7.

[15] J. Wibbeler, G. Pfeifer, and M. Hietschold, Parasitic charging of dielectric surfaces in capacitive microelectromechanical systems (MEMS), Sensors and Actuators, A 71, 1998, pp. 74-80.

[16] W.M. van Spengen, R. Puers, R. Mertens, and I. De Wolf, A comprehensive model to predict the charging and reliability of capacitive RF MEMS switches, J. Micromech. Microeng. 14, 2004, pp. 514-521.

[17] D.K. Schröder and J.A. Babcock, Negative Bias temperature instability: Road to cross in deep submicron silicon semiconductor manufacturing, J. Appl. Phys. 94, July 2003, pp. 1-18. 


\section{Authors' Biographies}

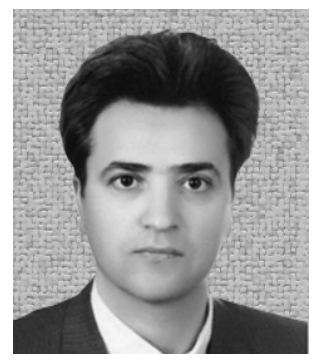

\section{Hamid REZA HOSEINI}

Hamid Reza Hoseini received his B.Sc. degree in E.E. from the Isfahan University of Technology (IUT), Isfahan, Iran, in 1992 and his M.Sc. degree in E.E. from the Islamic Azad University, Teheran, Iran, in 1996. He is currently pursuing his PhD degree in electronic engineering and his research is on shocked photonic crystals. He is also with Islamic Azad University, Arak Branch as an E.E. lecturer since 1996. Moreover he works with a project to get a MIS high efficiency photovoltaic solar cell.

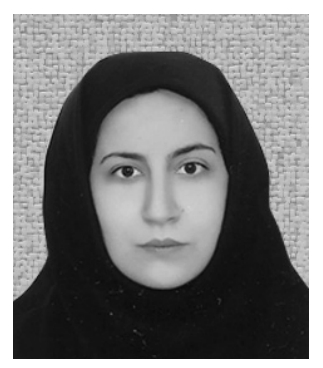

\section{Samaneh SADAT HASHEMIPOUR}

Samaneh S. Hashemipour received the B. Sc. degree in electronic engineering in 2006 from Azad University, Arak and the M.S degree in electronic engineering in 2009 from Azad University Arak. Her interests are in MEMS-based circuits.

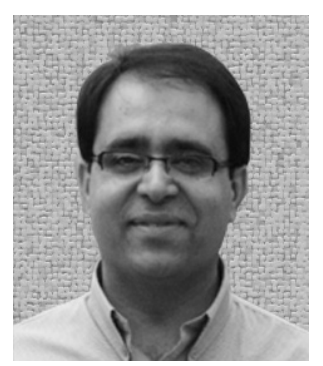

\section{Amir ABOLFAZL SURATGAR}

Amir Abolfazl Suratgar was born in Arak, Iran, in 1974. He received his B. S. degree in electrical engineering from Isfahan University of Technology, Isfahan, Iran, in 1996 with honors and his M. S. and Ph. D. degrees in control engineering from Amirkabir University of Technology (Tehran polytechnic), Tehran, Iran, in 1999 and 2002, respectively. He is assistant professor of electrical engineering at the University of Arak, Arak, Iran. Dr. Suratgar received the Outstanding Research Award from the engineering faculty of University of Arak in 2006 and 2005. He received the Outstanding EducationAward from engineering faculty of University of Arak in 2009. Currently he is with Amirkabir University of Technology (Tehran Polytechnic), Tehran, Iran. The area of his research interests are among system stability, system modeling and micro electro mechanical systems. He published more than 55 papers in scientific journals and conferences. 